\section{Letter to the Editor}

\section{Appropriate Research Training}

"Man is a key feature of development". This well founded assertion has inspired many organizations cooperating with the Third World. But results have not always satisfied the initial hopes. By funding grants without sufficiently clear conditions, actions were often useless and may have even generated difficulties.

Consider the specific problem of training, in the industrialized North, of students coming from the less advanced countries ( $L A C$ 's). A majority of them are working on programmes aiming at goals specific to industrialized countries. This leads one to ask:

- Is research conducted in the North by scientists from LAC's satisfying their countries' needs?

- Are scientists from LAC's being sufficiently encouraged to return home?

Some of the less developed countries have more scientists working in the North than at home. Expatriate scientists in fact often represent practically the only highlevel researchers available to some of these countries.

Meanwhile, research conducted by the North primarily aims at improving the quality of life, while that in the South is perhaps oriented towards survival. Is it impertinent to think that these two priorities are at least equal in value, and to hope that while some of the these Southern scientists carry on promoting "science", others could be allowed to pursue activities closely related to their countries' immediate and urgent needs?

If one-fourth of Southern scientists prepared their diplomas on topics directly related to development they would represent a remarkable potential for innovation in the Third World. But development of this resource should be managed with United Nations' support in a wide framework such as the European Community, and not in the narrow one of bilateral cooperation.

How can this potential be stimulated? By publicizing high priority topics which are vital for the South, and which could be pursued in laboratories in the North without a major shift in activities. This would be the task of data banks for research and development established jointly by North and South. The initiative should be complemented by a parallel programme of research grants for scientists.

And what can be done about the brain drain when many Southern scientists stay on after graduation in the country where they trained? It has various causes - but some actions would definitely make staying away from home less justifiable:

- Improved management of research carried out in the North:

- Definition of training programmes in terms of the real difficulties of Southerners: - Ensuring that the training of Southern scientists for high level diplomas forms only part of the function of Northern labora-

\title{
SOFIA's Objectives
}

(1) Interstellar cloud dynamics and star formation in our galaxy:

- Why and how do galactic clouds form stars?

- How important are magnetic fields and rotation for star formation?

(2) Proto-planetary disks and planet formation in nearby star systems:

- How common are solar systems? Under what conditions are they created?

(3) Origin and evolution of biogenic materials in the interstellar medium and in protoplanetary disks:

- What environments are hospitable to prebiotic molecules and compounds?

- What is the chemical composition of the interstellar medium?

(4) Comets, planetary atmospheres and rings in our solar system:

- How did our solar system evolve?

- What was the composition of the solar nebula?

(5) Star formation, dynamics and chemical content of other galaxies:

- How different are other galaxies?

- Why do some (e.g. colliding galaxies) exhibit extraordinarily large infrared luminosities?

-What is the origin of this luminosity?

- What is the structure and composition of the interstellar medium of other galaxies and the relation to star formation?

(6) The dynamic activity in the center of our own galaxy:

- What powers the highly luminous phenomena hidden at the center of the Milky Way - a compact star cluster or a black hole?

- Is this region similar to the "active galactic nuclei" seen in some other galaxies?

(7) The evolution of the Universe:

- What do very distant and very young galaxies look like?

- How do galaxies evolve?

- What is the form of the missing mass in the Universe?

-What lies beyond the galaxies?

\section{The Faculty of Science of the University of Fribourg, Switzerland}

has an opening for a

\section{FULL PROFESSOR IN THEORETICAL PHYSICS}

specifically in the fields of solid-state physics, statistical mechanics or field theory applied to condensed matter physics.

The successful candidate is expected to both reinforce and widen the current research activities at the Institute for Theoretical Physics (these comprise the theories of critical phenomena, superconductivity, correlated electron systems and surface physics). He will also have to teach both basic and advanced courses in theoretical physics, given in French and/or German.

It would be desirable if the new professor were disposed to interact with experimentalists of the Physics Institute. Two groups perform experiments in atomic and nuclear physics, one group works in solid-state physics.

Qualified applicants should submit their curriculum vitae, a statement of current research interests, a list of publications and the names of three professional references.

Applications should be sent to the: Dean of the Faculty of Science - University of Fribourg, Pérolles - CH-1700 Fribourg by September 15, 1990.

tories. The other part should consist in helping these scientists initiate or continue their normal professional activity in their own countries.

There already exists a "capital of research for development" in the North which should not be wasted but put to work using appropriate institutional structures and organizations. Meanwhile, we should worry about the continuing withdrawal of the best research teams in the North from cooperation with the South, a trend that is bound to increase due to the growing attractiveness of North-North cooperation on specifically Northern topics, and to the recent developments in eastern Europe.

\section{A. Kergreis}

Université de Paris XII, F-94010 Créteil 